\title{
ACOUSTIC DETECTIONS OF BLUE WHALE (BALAENOPTERA MUSCULUS) AND FIN WHALE (B. PHYSALUS) SOUNDS DURING A SURTASS LFA EXERCISE
}

BU- 1550-M

November 2000

\section{C.W. Clark \\ and}

Naomi S. Altman

Keywords: Acoustic detection, fin whales, LFA sonar, behavioral response, vocal behavior

\begin{abstract}
:
Acoustic data collected on the R/V Cory Chouest in February and March 1996 during active operation of the U.S. Navy's SURTASS LFA sonar were analyzed for blue whale (Balaenoptera musculus) and fin whale (B. physalus) sounds. Monitoring and mitigation protocols were implemented throughout the exercise to reduce the chances of an acoustic impact on marine mammals and sea turtles. Post-processed detections were compared with real-time detections to evaluate the effectiveness of the acoustic monitoring and mitigation process. Results indicate that there were cases in which Navy personnel missed acoustic detections of whales or misidentified non-biologic signals as whales. These missed detections were most likely because sounds were faint and probably from whales more than 3 nautical miles from the ship. There were insufficient detections of blue whales for further analysis. Fin whale acoustic detection probabilities were calculated from the post-processed data. Local linear regression analysis was used to compare fin whale detection probabilities from 1248 15-minute intervals under conditions when LFA sonar was and was not transmitting. There was a clear trend toward slightly higher fin whale sound occurrence during periods when there were no LFA transmissions. This trend may be the result of (a) reduced vocal activity by whales in response to LFA transmissions, (b) the effect of the mitigation protocols, or (c) some combination of (a) and (b). The data presently available cannot be used to distinguish among the alternatives, mainly because there were not enough data recorded for periods without LFA transmissions.
\end{abstract}




\title{
Acoustic Detections of Blue Whale (Balaenoptera musculus) and Fin Whale (B. physalus) Sounds During a SURTASS LFA Exercise
}

\begin{abstract}
Acoustic data collected on the RV Cory Chouest in February and March 1996 during active operation of the U.S. Navy's SURTASS LFA sonar were analyzed for blue whale (Balaenoptera musculus) and fin whale (B. physalus) sounds. Monitoring and mitigation protocols were implemented throughout the exercise to reduce the chances of an acoustic impact on marine mammals and sea turtles. Post-processed detections were compared with real-time detections to evaluate the effectiveness of the acoustic monitoring and mitigation process. Results indicate that there were cases in which Navy personnel missed acoustic detections of whales or misidentified non-biologic signals as whales. These missed detections were most likely because sounds were faint and probably from whales more than 3 nautical miles from the ship. There were insufficient detections of blue whales for further analysis. Fin whale acoustic detection probabilities were calculated from the post-processed data. Local linear regression analysis was used to compare fin whale detection probabilities from 1248 15-minute intervals under conditions when LFA sonar was and was not transmitting. There was a clear trend toward slightly higher fin whale sound occurrence during periods when there were no LFA transmissions. This trend may be the result of (a) reduced vocal activity by whales in response to LFA transmissions, (b) the effect of the mitigation protocols, or (c) some combination of (a) and (b). The data presently available cannot be used to distinguish among the alternatives, mainly because there were not enough data recorded for periods without LFA transmissions.
\end{abstract}

Index Terms -- Acoustic detection, fin whales, LFA sonar, behavioral response, vocal behavior

Manuscript received by November 2000. Grant N00014-96-1-0981 from the Office of Naval Research supported this work.

C. W. Clark is the I. P. Johnson Director of the Bioacoustics Research Program at the Cornell Laboratory of Ornithology, 159 Sapsucker Woods Rd., Ithaca, NY 14850, cwc2@,cornell.edu.

N. S. Altman is Associate Professor of Biometrics at Cornell University, Ithaca, NY 14850. 


\section{Clark and Altman -- LFA sonar and Whale Vocal Activity}

\section{INTRODUCTION}

Over the past decade, there has been an increased effort to understand the potential impact of humanmade underwater sounds on marine mammals $[1,2]$. This issue was first explicitly raised by Payne and Webb [3] who hypothesized that several species of whales produce low-frequency signals for very longrange communication. Specifically, two species of baleen whales within the balaenopterid group, blue (Balaenoptera musculus) and fin (B. physalus), produce long, patterned sequences of very intense ( $>180$ $\mathrm{dB}$ re $1 \mu \mathrm{Pa}$ at $1 \mathrm{~m})$, low-frequency $(15-35 \mathrm{~Hz})$ sounds that have excellent long-range transmission properties in the deep ocean [4], [5], [6], [7], [8], [9]. Payne and Webb [3] extended this hypothesis to suggest that low-frequency noise from modern ocean-scale shipping could seriously interfere with longrange communication, with an implied impact on individual reproductive success and survival. Recent empirical evidence supporting a long-range acoustic communication by blue and fin whales has been collected from Navy SOund SUrveillance System (SOSUS) hydrophone arrays and other bottom recorders [10], [11], [12], [13]. The specter of large-scale biological impact from human-produced lowfrequency underwater sounds remained buried until the advent of the Acoustic Thermometry of Ocean Climate (ATOC) oceanographic research project in 1993 [14]. Research on the effects of the ATOC sound on whales has revealed responses that were statistically significant but explain less than $5 \%$ of the total variation observed [15], [16]. The biological significance of these short-term, small-scale responses to a single sound source in terms of individual survival or species abundance remains uncertain but is suspected to be negligible.

During the last 10-15 years, in response to changes in submarine warfare technology, the US Navy has been developing and testing a new sonar system that relies on frequencies below $500 \mathrm{~Hz}$ to detect submarines [17]. This low-frequency sonar system is referred to as the SURveillance Towed Array Sensor System (SURTASS) Low Frequency Active (LFA) sonar. SURTASS LFA operates using a passive listening system and an active sonar system. The active sonar consists of a vertical array of 


\section{Clark and Altman -- LFA sonar and Whale Vocal Activity}

underwater transducers deployed from the center of the ship and the electronic hardware/software for controlling the operation of the sonar. The passive system consists of a hydrophone array deployed behind the ship and linked to specialized acoustic analysis workstations for detecting sonar echoes and discriminating targets. To date SURTASS LFA has operated on the R/V Cory Chouest and been tested during a number of exercises. Details of SURTASS LFA physical operations are beyond the scope of this paper but can be found in the Final Environmental Impact Statement (FEIS) (ref to Navy OEIS/EIS not yet available but will be soon?).

The results reported here were from an analyses of data collected during an SURTASS LFA sonar exercise. The exercise, referred to as LFA-15, was conducted on the R/V Cory Chouest in February and March 1996 off the coast of southern California. The primary objectives of the analyses were to 1) assess the reliability of Navy personnel at detecting and identifying low-frequency sounds of blue and fin whales, and 2) determine if there was any indication that whales changed their vocal behavior as a function of whether SURTASS LFA was or was not operating. The test and its accompanying monitoring and mitigation protocols were not designed to scientifically test the hypothesis that whales respond to SURTASS LFA sonar by changing vocal activity. Rather, the Navy agreed to implement the protocols in compliance with National Marine Fisheries Service (NMFS) recommendations. Here, for purposes of terminology, exercise refers to the LFA-15 operation, test refers to the 30 -minutes prior to and including a period of regular LFA sonar transmissions, and transmission refers to a single broadcast of the LFA sonar in the $100-500 \mathrm{~Hz}$ frequency band.

\section{METHODS}

\section{A. Mitigation and monitoring protocols}

Prior to the LFA-15 exercise an acoustic analysis workstation, referred to as Popeye, was installed aboard R/V Cory Chouest and Navy personnel were trained in its use. Four elements in the Cory's passive 


\section{Clark and Altman -- LFA sonar and Whale Vocal Activity}

hydrophone array were available for marine mammal data collection. These four hydrophones were spaced evenly along the array, approximately $500 \mathrm{~m}$ apart. Acoustic data from each hydrophone were low-pass filtered at $160 \mathrm{~Hz}$ prior to acquisition through analog-to-digital interface hardware at a sampling rate of $400 \mathrm{~Hz}$. This system allowed an operator to monitor a real-time, multi-channel sound spectrogram that displayed the sounds received on the four hydrophones from the Cory's towed passive array. The display scroll held the last hour of spectrographic data, and the monitor window displayed a userselectable, 10-minute segment of these data. Navy personnel were trained to recognize the most common types of species-specific sounds from baleen whales expected to occur in the LFA-15 operational area. Species recognition was based on comparison to spectrographic images of patterned sound sequences typical of blue, fin, and humpback (Megaptera novaeangliae) whales in the Northeastern Pacific. Estimation of a vocal whale's position relative to the Cory was based on computation of time-of-arrival differences of whale sounds on the different hydrophones using the Popeye software [18]. This location method does not account for refracted travel paths or bottom bounce, and assumes that propagation is dominated by the fundamental mode. This assumption probably is reasonable within a few miles of the ship, but not for greater distances.

Monitoring protocols specified that 30 minutes prior to the start of a test and throughout the test, Navy personnel would search the vicinity around the ship for marine mammals and sea turtles. Navy personnel used the Popeye system to monitor the passive acoustic array for the presence of whale sounds and to estimate the range to any whale detected. During daylight hours, Navy personnel visually monitored for the presence of marine animals around the ship using a pair of 25 power "big eye" binoculars. Mitigation protocols dictated that a) prior to the start of a test, the Navy would not begin transmitting LFA sonar if a marine mammal or sea turtle was detected within 3 nautical miles of the ship, and b) after the commencement of a test, transmissions would be suspended if a marine mammal or sea turtle was detected within 1 nautical mile of the ship. 


\section{Clark and Altman -- LFA sonar and Whale Vocal Activity}

\section{1) At-sea data collection}

For every 15-minute interval throughout a test, Navy personnel noted, by species, the estimated number of whales detected based on visual inspection of the real-time display. Identification of species was based on visual comparison to known species-specific spectrogram samples from the same region. The fourchannel acoustic data were multiplexed, and streamed to disk or removable data cartridge as a series of separate time-stamped files of a specified size. Most acoustic files were approximately 11 minutes long; a few were 17 minutes. After the completion of the exercise, the acoustic data files and hard copy detection logs were forwarded to Cornell for analysis.

\section{2) Post-processing}

Analysis of data logs and acoustic files focused on two questions. First, were the acoustic mitigation and monitoring protocols effective? Second, was there any evidence that whales changed their vocal behavior as a function of whether or not SURTASS LFA sonar was transmitting?

Blocks of time distributed throughout the exercise were selected for analysis. Each file within the selected blocks was opened and a spectrogram of the first channel (most forward hydrophone in the towed array) was examined. For each file, the number of blue whale sounds and fin whale sounds, referred to as a sound-count, and the estimated number of vocally active blue and fin whales, referred to as a whale-count, were noted. In most cases, the relative intensity of the highest-amplitude whale sound in the file was also noted using a subjective 4-point scale (1=very faint, $2=$ faint, $3=$ medium, 4=strong). Whale-count data were recorded both on a file-by-file basis and for successive 15 -minute intervals. A record was also kept of the presence or absence of "volcanic-blue" signals 1 in each file and 15-minute interval. To evaluate the effectiveness of acoustic monitoring and mitigation protocols, 15-minute interval

\footnotetext{
1"Volcanic blue" is the name given to a low-frequency signal of unknown origin that has been detected throughout the Pacific Ocean since the early 1990s. Some observers who recorded this signal suspected that it came from blue whales. Data
} 


\section{Clark and Altman -- LFA sonar and Whale Vocal Activity}

whale detections collected by Navy personnel in real-time were compared to the detections as notes by Cornell technicians during post-processing analysis.

Initially, the idea was to use sound-counts and whale-counts as a measure of acoustic activity. To evaluate this notion, 60 11-minute files from periods when Navy logs indicated relatively high numbers of whale acoustic detections were selected. Each file was examined separately by three research technicians experienced in acoustic detection of blue and fin whale sounds. These technicians recorded the number of blue and fin whale sounds (other species were not detected) and the loudness of the loudest sound. Comparison of these measures for the three analyst revealed that there was considerable variability in the number of sounds detected by each analyst, but there was good agreement on whether or not a species was detected. Therefore, only presence or absence of a species-specific whale sound was used for further analysis. The most likely explanation for the inter-analyst variability in sound-count values is that the majority of whale sounds were faint and not easily recognizable against the background noise. During this close examination of the files it was discovered that faint sounds were more difficult to detect in files containing a transmission. Therefore, files in which loudness was rated 1 (very faint) were not used in any further analysis.

\section{3) Performance evaluation}

To evaluate the performance of Navy personnel in acoustically detecting and identifying whales, whale-counts for the same 15-minute intervals as logged during the exercise were compared to counts as determined by Cornell analysts during post-processing. The time blocks selected for analysis contained a roughly equal mix of intervals when Navy personnel reported whales and intervals with no whales reported. The data were analyzed "blind" in that during the post-processing, Cornell analysts did not know what had been reported by Navy personnel. Data for blue and fin whales were treated separately.

presently available suggest that the signal, which appears to originate somewhere in the southern hemisphere near $54^{\circ} \mathrm{S}$, is not of biological origin. 
Clark and Altman -- LFA sonar and Whale Vocal Activity

For each species, each 15-minute interval was classified into one of the following three detection categories; correct, missed or false. "Correct" refers to the case in which Navy and Cornell personnel agreed that whale sounds were present in the interval. "Missed" refers to an interval for which Cornell personnel detected at least one whale and Navy personnel detected none. "False" refers to an interval for which Navy personnel detected one or more whales and Cornell personnel detected none. To generate a final performance assessment, the number of intervals was summed for each of the three categories above. Intervals for which both Navy and Cornell personnel reported no whales were excluded from performance evaluation analysis.

4) Comparison of fin whale acoustic detections during transmission-off and transmission-on periods

To evaluate the potential effect of LFA transmissions on whale vocal activity, data from periods with and without transmissions were compared. Inspection of Navy and Cornell sound-count data revealed that there were very few acoustic detections of blue whales. Therefore, only data on fin whales were used in the comparative analysis of vocal activity during periods with and without transmissions. LFA-15 exercise protocols did not specifically include the collection of control data during periods without transmissions. However, there were a few periods of at least $1 \mathrm{~h}$ duration throughout the exercise when there were no transmissions and for which acoustic files were available. Such periods occurred either as a result of difficulties with LFA equipment or because a whale was detected near the vessel and transmissions were suspended. These periods with no transmissions will be referred to as transmission-off periods. Periods during which transmissions occurred will be referred to as transmission-on periods. For each file analyzed from transmission-on and transmission-off periods, the transmission condition, start time (in decimal hours from the start of the exercise), detection condition $(0=$ no fin sound detected; $1=$ fin sound(s) detected), sound-counts, and the relative loudness of the loudest fin whale sound were noted. 


\section{Clark and Altman -- LFA sonar and Whale Vocal Activity}

Since sound-count measures were not deemed reliable, detection condition was used as the measure of acoustic response, and the data were analyzed in terms of detection probability. If the probability of detecting a whale sound were constant throughout the exercise, the estimated detection probability would be the mean of the binary outcomes of the detection condition. More realistically, for a given transmitting state ("on" vs. "off"), the probability of detection was expected to vary with time due to whale activity and ship movement. Accordingly, the probability of detecting a whale sound within a given time interval, with transmission either on or off, was approximated by a weighted average of the detection condition in neighboring intervals

We used local linear regression to evaluate detection probabilities over the time period of the experiment. Local linear regression has been shown to have excellent statistical properties for local averaging [19], [20]. Aragaki and Altman [21] have shown that the technique can be applied to estimate the probability of a " 1 " for binary response. Therefore, to estimate the probability of detecting a sound at time $\mathrm{T}$, a neighborhood of $\mathrm{T}$ was selected. A weighted linear regression of the binary response against time was fitted in the neighborhood. The estimated probability was the fitted value at T. A standard error for the estimate was computed from standard techniques of weighted least squares regression.

Because a linear regression is not constrained to be between 0 and 1 , local linear regression can give estimates of the sound detection probability that are outside the plausible range. This should not happen for data rich periods, but may occur particularly at the edges of gaps in the data record. Aragaki and Altman [21] have shown that truncating the estimates at the boundaries gives a reasonable estimate in this case.

Appropriate choice of neighborhood size (span) is critical for good performance of local linear regression. Altman and MacGibbon [22] and Aragaki and Altman [21] demonstrated that commonly used automatic techniques for selecting span do not work well for binary response data. Accordingly, span was 


\section{Clark and Altman -- LFA sonar and Whale Vocal Activity}

chosen subjectively by one of us (NSA) from a range of plausible spans for the February data, and then adjusted for sample size for the March data. For the February data, interpretation did not vary much with choice of span; the span was selected primarily for clarity.

The S-plus routine LOESS was used to provide the local linear fit and confidence bands [23]. LOESS uses the average variance of the data in computing the bands. For binary data with probability $\mathrm{P}$ of "1", the variance is $\mathrm{P}(1-\mathrm{P})$. Therefore, the plotted bands are too wide at high and low detection probabilities, and too narrow when the detection probability is close to $50 \%$.

Autocorrelation in the data over time may be induced by a single whale vocalizing over a lengthy period, or by animals vocalizing in response to the sounds of other animals. Autocorrelation does not affect the efficacy of local linear regression for estimating detection probability if a suitable span is chosen, but it does make the confidence bands wider [24]. In the analysis presented here, the displayed confidence bands were not adjusted for the possible effects of autocorrelation.

To assess whether or not there is a statistically significant effect of LFA transmissions on whale vocal activity, we selected the 34 time periods during which there were 4 or more consecutive files with no pings followed by 4 or more consecutive files with pings. We then used paired t-tests to compare call rates in these periods. To eliminate possible effects of the mitigation protocol on these results, the analysis was repeated for the subset of 20 time periods which did not include any vocalizations rated as "loud".

\section{RESULTS}




\section{Clark and Altman -- LFA sonar and Whale Vocal Activity}

\section{B. Performance evaluation}

In total there were 1248 15-minute intervals compared, 808 (202 h) from February and 440 (110 h) from March 1996. Patterned sequences of blue and fin whale sounds were detected in both months along with volcanic-blue sounds, as shown in Figure 1. It is important to note that the post-processed spectrograms examined by Cornell personnel were higher in resolution and larger on the monitor than spectrograms viewed by Navy personnel monitoring sounds in real-time. This difference is illustrated in Figure 2. As a result of these differences, Cornell technicians had an advantage over Navy personnel in detecting faint signals with a low signal-to-noise ratio.

Tables 1 and 2 and Figure 3 summarize the results of the performance evaluation comparing blue and fin whale detections noted by Navy personnel in real-time and Cornell technicians during post-processing.

\section{1) Blue whales.}

Detection of blue whale signals was low in both months. For February, Navy personnel missed detecting blue whales in all 41 of the intervals in which Cornell technicians detected them. All of the 44 intervals with blue whale detections reported by Navy personnel turned out to have no blue whales in them according to Cornell technicians. However, $31(70 \%)$ of the intervals with false blue whale detections did contain volcanic-blue signals. In all likelihood, these were the signals misidentified as blue whales by Navy personnel.

For March, there were no blue whale detections by the Navy while Cornell personnel detected blue whales in 15 of the 440 intervals.

\section{2) Fin whales.}

There were considerably more detections of fin whale sounds than blue whale sounds in both months. For all 1248 intervals from both months, Navy personnel detections were correct for $64 \%$ of the intervals and false for $17 \%$ of the intervals. 


\section{Clark and Altman -- LFA sonar and Whale Vocal Activity}

\section{Effects of LFA on whale vocal behavior}

386 hours from a total of 655 hours of available acoustic files from the LFA-15 exercise were analyzed. Figures 4 shows the transmission schedule and the availability of archived acoustic data for the exercise. Figures 5 and 6 plot confidence intervals for the probability of detecting a fin whale sound as a function of time for the transmission-on (light gray band) and transmission-off (dark gray) conditions for February and March, respectively. In these figures, each 11-minute data file that was analyzed is represented by a black dot. If there are no black dots in the confidence band then there are either no data available or the 11-minute files were not analyzed. Sound detection data for transmission-on and transmission-off conditions were smoothed separately - hence rates in one condition do not affect the estimates in the other condition.

One can see in Figure 5 that when the sound detection probability is low (e.g., around 100 hours, and again around 270 hours), there is little difference in the probability of fin sound detection between the transmission-on and transmission-off conditions. However, when the probability of a fin sound detection is high (e.g., around 50, 200, and 325 hours), it is higher during transmission-off periods. This difference in sound probability for the "on" vs. "off" condition is indicated by the offset between the light gray and dark gray bands. These shaded regions can be thought of as error bars and will be referred to as error bands. The dashed lines connecting the upper and lower edges of the bands for successive transmissionon periods and successive transmission-off periods are the outlines of the error band for estimated sound probabilities between these periods.

In many cases the sound probabilities for adjacent transmission-on and transmission-off periods are obviously different. This is especially true if the detection probability is high (e.g., high vocal activity). For example, in March (Figure 6) there is an abrupt increase in detection probability when transmissions are suspended around the 72 hour position. Differences in detection probability are not as obvious different when detection probability is low (e.g., low vocal activity). For example, in March (Figure 6) at 


\section{Clark and Altman -- LFA sonar and Whale Vocal Activity}

285 hours into the test, after the LFA had been transmitting regularly at about 18 hours and the detection probability is low, there is no obviously large change during the approximately 1 hour period without transmissions.

Plotted detection probabilities may provide a misleading picture, however. Of the 34 time periods in February with at least 4 consecutive files without pings followed by 4 consecutive files with pings, 17 had a higher mean call rate and 13 had a lower mean call rate with transmission-off. The paired $t$-test for the difference in rates is not significant $(p=0.48)$. Fourteen of these time periods contained loud whale vocalizations which indicate whales close to the vessels. Of the remaining 20 time periods, 8 had a higher mean call rate and 8 had a lower mean call rate with transmission-off. The paired $t$-test for the difference in call rates is not significant $(p=0.43)$.

\section{DISCUSSION}

\section{Discrepancies in detections}

Most of the blue whale detections reported by Navy personnel appear to be misidentified volcanicblue signals. Confusion about discriminating between these two signal types may also be the cause of many of the missed detections (i.e., real blue whale sounds were overlooked because they did not look enough like volcanic-blues).

The discrepancies between Navy and Cornell detections of fin whale sounds have several explanations. Fin whale sounds are about $1 \mathrm{~s}$ in duration. When received as low intensity signals they are easily confused with transient ambient noise pulses. Some of the missed detections may have been more obvious to Cornell than to Navy personnel because of the higher resolution and larger size of the spectrogram displays used in post-processing (Figure 2). 


\section{Clark and Altman -- LFA sonar and Whale Vocal Activity}

The apparent detection and identification errors probably had little or no impact on compliance with the mitigation protocol because most of the whale sounds that were missed were fairly weak sounds, hence probably from distant animals well beyond the 3 nautical mile mitigation radius. For example, for the blue whale sound detections that were missed by Navy personnel, usually only two or three sounds were detected during post-processing, they occurred in a period of 3-5 minutes, and all were very faint. This suggests that these sounds were from animals in the first convergence zone approximately 25-30 miles away. However, complete failure to correctly identify a sound type (e.g., blue whales) could in the future cause an operator on the Cory to overlook a sound from a nearby animal. This in turn could lead to a violation of the mitigation protocol if transmissions were not suspended.

Detection performance for both blue and fin whale signals could undoubtedly be significantly improved with further training of Navy personnel and better acoustic display options for transient signals.

\section{E. Potential Behavioral Response}

There is some evidence to suggest that fin whales responded to LFA sonar transmission by decreasing their vocal activity. This result is confounded by the LFA protocol that required that transmissions be suspended when a whale was located within $1 \mathrm{nmi}$ of the ship. Clearly, if transmissions were suspended only when whales were detected near the vessel, whale sounds would be more prevalent during off periods. Since the logs available to Cornell were not sufficiently detailed to determine in all cases whether a particular suspension was because of a whale contact, only the times of sounds loud enough to yield a reliable location were plotted. The vertical bars in Figures 5 and 6 depict the times of relatively strong (solid lines) and medium (dashed lines) sounds in the data files. If intensity was not noted, a dashed line was plotted. In the March data, for example, one can see that medium level sounds occurred at 72 hours, and transmissions were suspended. By contrast, a number of medium level sounds were detected after 120 hours, when transmissions had already been off for a few hours. At about 138 hours, transmissions resumed and this may have been due to the location of the whale(s) making the unrated 


\section{Clark and Altman -- LFA sonar and Whale Vocal Activity}

sounds just prior to this time. Suspensions that were not immediately preceded by strong or mediumintensity sounds were probably not due to detection of a whale near the vessel. The data provide little evidence that whales were more likely to vocalize when the Cory was not transmitting.

\section{E. Potential Behavioral Response and Biological Impact}

What do these results suggest with regard to the potential impact of LFA sonar sounds on baleen whales? In the case presented here, where the response measure was a change in vocal activity, the answer requires some knowledge of the biological significance of the vocal activity. Unfortunately, there is not much information on the biological function or natural variation of fin whale sounds. It is assumed that the long, patterned sounds as detected here, are male reproductive displays [9]. It is also assumed that fin whales in the Northern Hemisphere are engaged in breeding in February and March [25]. Thus, a reasonable working basis is that these sounds are male reproductive displays that advertise something about a male's quality or breeding status. Therefore, by extension, disruption of this display behavior could change a male's breeding success. Under natural circumstances, within any given geographic area, singing activity appears to vary considerably in terms of a number of acoustic parameters including song duration, number of songs in a singing bout, and sound level [8], [9]. Thus, for example, Watkins et al. [9] found statistically significant variation in song bout length over the year, while Curtis et al. [26] show evidence of variation in fin whale vocal activity as a function of latitude.

Miller et al. [27] reported a $29 \%$ increase in humpback song duration for singers during experimental exposure to LFA sonar, although these results were confounded by the presence of the observation vessel. Humpback whales have been observed to respond to other types of low-frequency human-made sounds (e.g., small vessel noise) by changing singing behavior [28], avoiding the area of the sound source [29], or changing surfacing behavior [15], [16]. 


\section{Clark and Altman -- LFA sonar and Whale Vocal Activity}

Gray whales (Eschrichtius robustus) migrating of California are known to respond to various industrial noises from oil and gas activities and to LFA sonar by swimming around the area of the source [30], [2]. When an LFA sound source was placed in the central migratory corridor, the level of response graded with received level, suggesting that received level was the primary cue to which animals were responding. However, when the LFA source was moved offshore, no avoidance response was detected even when received levels were equal to those for animals in the central migratory corridor [31]. Bowhead whales (Balaena mysticetus) show a very strong respond to seismic impulses from oil and gas exploration activities and were rarely seen within $20 \mathrm{~km}$ of the seismic, airgun array source [32], [33]..

Overall there is evidence from a number of different baleen species and for a number of different sound sources that whales respond to low-frequency human-made sounds. The response was sometimes obvious, as in the case when a sound source was placed within the central corridor of migrating gray whales. Most often, for example during breeding or feeding contexts, the response has been subtle and explains only a small percentage of observed variation. In all cases so far, the response has been shortlived. Migrating gray whales appear to return to their normal migratory route within a few miles after the offending sound source. Singing humpback whales resume singing and engage in normal social activities within tens of minutes following the response. In the case presented here, the change in the aggregate vocal activity of fin whales appears to be relatively small and short-lived.

Interpretation of these results in terms of potential biological impact is difficult. The proportion of the breeding population affected can not be determined. All the evidence suggests that relatively few breeding males were within detection range of the LFA sonar. In over $90 \%$ of the cases when fin whales were detected only one animal was heard. Detection rate was relatively modest (63\%, see Fig. 3$)$ and the percentage of sounds subjectively rated as loud was small $(<5 \%)$. Interpretation of any changes in vocal behavior remains speculative since we do not yet know enough about the mechanisms of vocal communication in these whales to evaluate the reproductive cost to a male who changes his singing 


\section{Clark and Altman -- LFA sonar and Whale Vocal Activity}

behavior in response to low-frequency sound. In the case of the acoustic data available here, it was not possible to reliably measure song characteristics (e.g. song duration, bout duration) because very few if any animals were close enough for accurate measurements.

In cases where further measurements have been available in the presence and absence of loud lowfrequency sound, changes in whale vocal behavior have been either inconsistent, or relatively small and the low-frequency sound condition explained only a small fraction of the measured variability. For example in 1997 during research on LFA sonar, Croll et al. [10] reported that fin whale vocal activity measured at one location increased significantly from experimental to the post-experimental period, but decreased significantly at a second location. Their conclusion was that these changes were more indicative of relative distance of whales to food resources than to the LFA sonar. Unfortunately the data were too sparse to remove the influence of prey distribution to statistically test for the influence of the LFA sonar. For humpbacks off Kauai, Frankel and Clark [16] found statistically significant differences in time and distance between surfacings between blocks of time with control (ATOC-off) and experimental (ATOC-on) conditions. However, the ATOC condition explained less than $5 \%$ of the total variation in these measures of surface behavior, and the authors concluded that the responses of humpback singers to the ATOC sound were probably not biologically significant.

In the case of the data reported here for an LFA sonar exercise, we note that both the level of vocal activity and the sound levels of the received fin whale sounds were relatively low. There was a definite tendency to detect less fin whale vocal activity to decrease during operation of the LFA sonar, but the lack of control data make it impossible to test for the significance of any differences in the measured acoustic behavior, especially since detection of loud calls was a determinant in stopping LFA transmissions. Without proper control and experimental data, and without a better understanding of fin whale singing, the biological significance of any response, whether a small-scale behavioral change or a long-term change in breeding success, or potential impacts from cumulative exposures to a suite of 


\section{Clark and Altman -- LFA sonar and Whale Vocal Activity}

human-made low-frequency noises, remains speculative. At this point it appears that exposure to LFA sonar does not lead to an immediately observable change in vocal activity and that the behavioral changes that can be measured are small relative to natural variability.

The biological significance of these short-term, small-scale responses to a single sound source in terms of individual survival or species abundance remains uncertain but is suspected to be negligible. However, in the long-term it is the cumulative impact of multiple sources, not impact from just one source, that is the major concern. It is not unreasonable to suspect that the additive effect of many loud, low-frequency acoustic events from a variety of sources reduces the availability of breeding habitat, changes migratory routes, or interferes with breeding displays in ways too subtle to be detected by smallscale or short-term studies. There is no doubt that the industrialization of coastal areas in support of commercial shipping has seriously reduced available habitat for coastal species (e.g., gray whales and northern right whales). In the short-term, this alone does not predict their demise, as witnessed by the recovery of the Eastern Pacific gray whale population. However, serious consideration of such environmental concerns must go beyond the limits of the human memory. Many of these speculated impacts, whether from noise, heavy metals, or organic compounds, are not testable and are beyond the scope of scientific manipulation. Oversight, caution, common sense, and full consideration of an ecological approach must be combined with the best available scientific evidence to reach a responsible solution for environmental conservation. In the case of dealing with specific types of potentially biologically harmful sound sources, it would seem most prudent to combine mitigation and monitoring, research, and regular evaluation as a means of controlling for impact.

\section{Acknowledgements}

This work was supported by a grant N00014-96-1-0981 from ONR. This research would not have been initiated without the support of Dennis Conlon and Robert Gisiner, and would not have been 


\section{Clark and Altman -- LFA sonar and Whale Vocal Activity}

accomplished without the cooperation and enthusiastic help from the crew and Navy personnel aboard the R/V Cory Chouest. For the post-processing effort, we particularly thank Carol Carson, Katherine Dunsmore and Melissa Fowler for acoustic analysis; Katherine Dunsmore and Kurt Fristrup were responsible for the S-Plus programming and plotting; Russ Charif completed much of the detection evaluation analysis. 


\section{Clark and Altman -- LFA sonar and Whale Vocal Activity}

\section{LITERATURE CITED}

[1] National Research Council (NRC), Low-frequency sound and marine mammals: Current knowledge and research needs, D. M. Green, H. A. Ferrarri, D. McFadden, J. S. Pearse, A. N. Popper, W. J. Richardson, S. H. Ridgway and P. L. Tyack, Eds. National Academy Press, Washington, D.C. 1994.

[2] W. J. Richardson, C. R. Greene, Jr., C. I. Malme, and D. H. Thomson, Marine Mammals and Noise. Academic Press, New York, 1995.

[3] R. Payne and D. Webb, "Orientation by means of long range acoustical signaling in baleen whales," Annals of the New York Acad. Sci., vol. 188, pp. 110-141, 1971.

[4] C. W. Clark and W. T. Ellison, "Potential use of low-frequency sounds by baleen whales for probing the environment: evidence from models and empirical measurements," in Echolocation in Bats and Dolphins, J. Thomas, C. Moss and M. Vater, Eds. The University of Chicago Press. In press.

[5] C. W. Clark and K. M. Fristrup, "Whales '95: A combined visual and acoustical survey of blue and fin whales off southern California," Rep. int. Whal. Commn., vol. 47, pp. 583-600, 1997.

[6] W. C. Cummings and P. O. Thompson, "Underwater sounds from the blue whale, Balaenoptera musculus," J. Acoust. Soc. Amer.., vol. 50, pp. 1193-1198, 1971.

[7] J. A. Rivers, "Blue whale, Balaenoptera musculus, vocalizations from the waters off central California," Mar. Mamm. Sci., vol. 13, pp. 186-195, 1997.

[8] P. O. Thompson, L. T. Findley, and O. Vidal, " $20-\mathrm{Hz}$ pulses and other vocalizations of fin whales, Balaenoptera physalus, in the Gulf of California, Mexico," J. Acoust. Soc. Amer., vol. 92, pp. 30513057, 1992.

[9] W. A. Watkins, P. Tyack, K. E. Moore, and J. E. Bird, "The 20-Hz signals of finback whales (Balaenoptera physalus)," J. Acoust. Soc. Amer., vol. 82, pp. 1901-1912, 1987.

[10] D. A. Croll, C. W. Clark, J. Calambokidis, W. T. Ellison, and B. R. Tershy, "Effect of anthropogenic low frequency noise on the foraging ecology of Balaenoptera whales," Anim. Conserv., in press.

[11] M. A. McDonald, J. A. Hildebrand, and S. C. Webb, "Blue and fin whales observed on a seafloor array in the northeast Pacific," J. Acoust. Soc. Amer., vol. 98, pp. 712-721, 1995.

[12] K. M. Stafford, C. G. Fox, and D. S Clark, "Long-range acoustic detection and localization of blue whale calls in the northeast Pacific Ocean," J. Acoust. Soc. Amer., vol. 104, pp. 3616-3625, 1998.

[13] W. A. Watkins, M. A. Daher, G. M. Reppucci, J. E. George, D. L. Martin, N. A. DiMarzio, and D. P. Gannon, "Seasonality and distribution of whale calls in the North Pacific," Oceanography, vol. 13, pp. $62-67,2000$. 


\section{Clark and Altman -- LFA sonar and Whale Vocal Activity}

[14] W. Munk, "The sound of oceans warming," The Sciences, September/October, pp. 21-26, 1993.

[15] A. S. Frankel and C. W. Clark, "Results of low-frequency m-sequence noise playbacks to humpback whales in Hawai'i," Can. J. Zool., vol. 76, pp. 521-535, 1998.

[16] A. S. Frankel and C. W. Clark. "Behavioral responses of humpback whales (Megaptera novaeangliae) to full-scale ATOC signals," J. Acoust. Soc. Amer., vol. 108, pp. 1930-1937, 2000.

[17] G. D. Tyler, Jr., "The emergence of low-frequency active acoustics as a critical antisubmarine warfare technology," Johns Hopkins APL Technical Digest, vol. 13, pp. 145-159, 1992.

[18] C. W. Clark and W. T. Ellison, "Calibration and comparison of the acoustical location methods used during the spring migration of the bowhead whale, Balaena mysticetus, off Pt. Barrow, Alaska, 19841993," J. Acoust. Soc. Amer., vol. 106, pp. 3509-3517. 2000.

[19] W. S. Cleveland and S. J. Devlin, "Locally weighted regression: an approach to regression analysis by local fitting," J. Amer. Stat. Assoc., vol. 83, pp. 596-610, 1988.

[20] J. Fan, "Design-adaptive nonparametric regression," J. Amer. Stat. Assoc., vol. 87, pp. 998-1004, 1992.

[21] A. Aragaki and N. S. Altman, "Local polynomial regression for binary response," Biometrics Unit Technical Report, BU-1397-M, 1997.

[22] N. S. Altman and B. McGibbon, "Consistent bandwidth selection for kernel binary regression," J. Stat. Plan. and Infer., submitted for publication.

[23] Mathsoft, S-Plus 2000: Modern Statistics and Advanced Graphics. Data Analysis Products Division, Mathsoft, Inc. Seattle, WA, 1999.

[24] N. S. Altman. "Kernel smoothing of data with correlated errors," J. Amer. Stat. Assoc., vol. 85, pp. 749-759, 1990.

[25] K. S. Norris, Whales, Dolphins, and Porpoises. University of California Press, Berkeley, CA, 1966.

[26] K. R. Curtis, B. M. Howe, and J. A. Mercer, "Low-frequency ambient sounds in the North Pacific: long time series observations," J. Acoust. Soc. Amer., vol. 106, pp. 3189-3200, 1999.

[27] P. J. O. Miller, N. Biassoni, A. Samuels, and P. L. Tyack, "Whale songs lengthen in response to sonar," Nature, vol. 405, p. 903, 2000.

[28] T. F. Norris, "Effects of boat noise on the acoustic behavior of humpback whales," J. Acoust. Soc. Amer., vol. 96, p. 3251, 1994.

[29] J. Calambokidis, T. E. Chandler, D. P. Costa, C. W. Clark, and H. Whitehead, "Effects of ATOC sound source on the distribution of marine mammals observed from aerial surveys off central 


\section{Clark and Altman -- LFA sonar and Whale Vocal Activity}

California," in Abstracts of the World Marine Mammal Science Conference, Monaco, 24-24 January 1998. Society for Marine Mammalogy, Lawrence, KA, 1998, p. 22.

[30] J. R. Buck and P. L. Tyack, "Response of gray whales to low-frequency sounds," J. Acoust. Soc. Amer., vol.107, p.2774, 2000.

[31] P. L. Tyack and C. W. Clark, "Quicklook -- Playback of low frequency sound to gray whales migrating past the central California coast - January, 1998," unpublished technical report, 1998.

[32] Richardson, W.J. (ed). 1999. Marine mammal and acoustical monitoring of Western Geophysical's open-water seismic program in the Alaskan Beaufort Sea, 1998. LGL Rep. TA2230-3. Rep. from LGL Ltd., King City, Ont., and Greeneridge Sciences Inc., Santa Barbara, CA, for Western Geophysical, Houston, TX, and U.S. Nat. Mar. Fish. Serv., Anchorage, AK, and Silver Spring, MD. $390 \mathrm{p}$.

[33] Richardson, W.J., G.W. Miller and C.R. Greene Jr. 1999. Displacement of migrating bowhead whales by sounds from seismic surveys in shallow waters of the Beaufort Sea. J. Acoust. Soc. Am. 106(4, Pt. 2):2281. 


\section{Clark and Altman -- LFA sonar and Whale Vocal Activity}

\section{Figure Captions}

Figure 1. Example spectrograms showing (a) "volcanic blue" signature, a sound originating in the southern hemisphere and believed to be a result of a geophysical event, (b) blue whale sounds typical of the Northeastern Pacific region, and (c) fin whale "20-Hz" pulses.

Figure 2. Comparison of spectrogram displays of the same data as shown by (a) the real-time browser monitored by Navy personnel, and (b) a Popeye spectrogram of the type examined by Cornell technicians during post-processing. The multi-channel browser display shows a frequency range of 5 $100 \mathrm{~Hz}$; the single-channel spectrogram shows $15-45 \mathrm{~Hz}$. Both displays show 275 seconds of data. Faint fin whale calls (rapid down sweeps in 17 - $40 \mathrm{~Hz}$ range) may be more detectable in the postprocessing display.

Figure 3. Blue whale detections (a) by Navy personnel, February - March 1996 (LFA-15). Same data as in Table 1. Percentage values are relative to the total analyzed sample of 1248 intervals. "None" indicates intervals in which neither Navy nor Cornell personnel detected whale calls. There were zero correct detections. Finback whale detections (b) by Navy personnel, February - March 1996 (LFA15). Same data as in Table 2. Labeling as in Figure 2.

Figure 4. Transmission and tape schedule from (a) February and (b) March, 1996. '+' symbols indicate times of transmissions; horizontal lines indicate times when archived acoustic data were available.

Figure 5. Probability of fin whale call occurrence for the transmission-off (light gray) and transmission-on (dark gray) conditions, beginning at $1327 Z$ on 3 February 96 . Bands are \pm 1 standard error wide. Dots in middle of bands indicate start times of the 11-minute files analyzed. Dashed lines connecting probability bands in successive transmission-on or transmission-off periods indicate interpolated confidence intervals. Vertical lines indicate times of whale calls: solid line $=$ strong call, dashed line $=$ medium call, dotted line = unrated call. See text for further explanation. (Figure continues on next page.)

Figure 6. Probability of fin whale call occurrence for the transmission-off (light gray) and transmission-on (dark gray) conditions, beginning at $1502 Z$ on 5 March 96. Symbols as in Figure 5. 